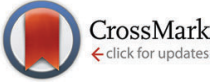

Cite this: Phys. Chem. Chem. Phys., $2015,17,24944$

Received 18th July 2015 Accepted 24th August 2015

DOI: $10.1039 / c 5 c p 04214 c$

www.rsc.org/pccp

\section{Structure, solvent, and relativistic effects on the NMR chemical shifts in square-planar transition- metal complexes: assessment of DFT approaches $\dagger$}

\author{
Jan Vicha, $\ddagger^{\mathrm{ab}}$ Jan Novotný, $\dot{\ddagger}^{\mathrm{a}}$ Michal Straka, ${ }^{\text {ac }}$ Michal Repisky, ${ }^{\mathrm{d}}$ Kenneth Ruud, ${ }^{d}$ \\ Stanislav Komorovsky*d and Radek Marek*ae
}

\begin{abstract}
The role of various factors (structure, solvent, and relativistic treatment) was evaluated for square-planar $4 d$ and $5 \mathrm{~d}$ transition-metal complexes. The DFT method for calculating the structures was calibrated using a cluster approach and compared to X-ray geometries, with the PBEO functional (def2-TZVPP basis set) providing the best results, followed closely by the hybrid TPSSH and the MN12SX functionals. Calculations of the NMR chemical shifts using the two-component (2c, Zeroth-Order Regular Approximation as implemented in the ADF package) and four-component (4c, Dirac-Coulomb as implemented in the ReSpect code) relativistic approaches were performed to analyze and demonstrate the importance of solvent corrections (2c) as well as a proper treatment of relativistic effects (4c). The importance of increased exact-exchange admixture in the functional (here PBE0) for reproducing the experimental data using the current implementation of the $2 \mathrm{c}$ approach is partly rationalized as a compensation for the missing exchange-correlation response kernel. The kernel contribution was identified to be about $15-20 \%$ of the spin-orbit-induced NMR chemical shift, $\Delta \delta_{\mathrm{SO}}$, which roughly corresponds to an increase in $\Delta \delta_{\mathrm{SO}}$ introduced by the artificially increased exact-exchange admixture in the functional. Finally, the role of individual effects (geometry, solvent, relativity) in the NMR chemical shift is discussed in selected complexes. Although a fully relativistic DFT approach is still awaiting the implementation of GIAOs for hybrid functionals and an implicit solvent model, it nevertheless provides reliable NMR chemical shift data at an affordable computational cost. It is expected to outperform the 2c approach, in particular for the calculation of NMR parameters in heavy-element compounds.
\end{abstract}

${ }^{a}$ CEITEC - Central European Institute of Technology, Masaryk University, Kamenice 5/A4, CZ-62500 Brno, Czech Republic. E-mail: radek.marek@ceitec.muni.cz

${ }^{b}$ Centre of Polymer Systems, University Institute, Tomas Bata University in Zlin, Trida T. Bati 5678, CZ-76001 Zlin, Czech Republic

${ }^{c}$ Institute of Organic Chemistry and Biochemistry, Academy of Sciences of the Czech Republic, Flemingovo nám. 2, CZ-16610 Prague, Czech Republic

${ }^{d}$ Centre for Theoretical and Computational Chemistry, Department of Chemistry, UiT - The Arctic University of Norway, N-9037 Tromsø, Norway.

E-mail: stanislav.komorovsky@uit.no

${ }^{e}$ Department of Chemistry, Faculty of Science, Masaryk University, Kamenice 5, CZ-62500 Brno, Czech Republic

$\dagger$ Electronic supplementary information (ESI) available. Fig. S1: role of dispersion (empirical D3 correction) in cluster-based geometry optimizations; Fig. S2: the RMSDs (pm) for the interatomic distances according to the type of central metal; Fig. S3: the RMSDs (pm) for the interatomic distances according to the type of the light spectator atom; Fig. S4: comparison of total RMSDs (in pm) for interatomic distances calculated in vacuo and in cluster; Fig. S5: the 4c mDKS NMR chemical shifts with and without empirical "CGO corrections". Table S1: the $\Delta \delta(\mathrm{ppm})$, in vacuo; Table S2: the $\Delta \delta$ (ppm), COSMO; Table S3: the $\Delta \delta_{\mathrm{kernel}}^{\mathrm{XC}}$ values (ppm). See DOI: $10.1039 / \mathrm{c} 5 \mathrm{cp} 04214 \mathrm{c}$

\$ These two authors contributed equally.

\section{Introduction}

Square-planar transition-metal complexes, molecules with ligands arranged in a square-like pattern around the central metal, are typically preferred by $\mathrm{d}^{8}$ elements such as platinum(II). Their unique chemistry is dominated by a trans effect that makes them well suited for various practical applications ranging from cancer treatment (cisplatin, oxaliplatin) ${ }^{1}$ to chemical catalysis, ${ }^{2}$ molecular switches and beyond. ${ }^{3}$ Generally, a detailed analysis of the structure and various properties is required to understand their biological effects in the human body as well as their role in catalytic processes. In addition to experimental methods (e.g., X-ray diffraction, NMR spectroscopy) theoretical methods are also frequently being employed to investigate their structure, chemistry and properties, often supplementing experimental NMR studies. The synergy between theoretical and experimental approaches allows us to combine the experimental precision with the predictive and explanatory power of theoretical methods, yielding new insight unachievable by the experimental or theoretical approaches alone. ${ }^{4}$ Relativistic effects 
have a significant influence on the NMR chemical shifts of light ligand atoms (LA) in the vicinity of the heavy-atom center (HA). Particular attention in NMR calculations should be paid to the spin-orbit effects, part of so-called HALA effects, ${ }^{5}$ typically propagated from the heavy element to light spectator atoms via the spin-orbit/Fermi-contact (SO/FC) mechanism. ${ }^{5,6}$ The spin-orbit contribution to the NMR chemical shift, $\Delta \delta_{\text {So }}$, represents a relativistic correction to the NMR chemical shift that can amount from ten to hundreds of ppm. ${ }^{7}$

Calculated relativistic effects in the vicinity of the heavy metal are generally very sensitive to (i) the character of metal-ligand bonding, ${ }^{8,9}$ requiring the use of correct and accurate structures, (ii) the inclusion of environmental effects (e.g., solvent), ${ }^{10}$ and (iii) reliable methods for treating the relativistic effects. ${ }^{11}$

(i) Density functional theory (DFT) is nowadays the most commonly used method for optimizing the structures and calculating various properties of molecules and molecular systems, mainly due to its favorable scaling with system size and the rather good accuracy that can be achieved. A large number of density functionals have been developed over the years for calculating the molecular structures and/or molecular properties. The performance of individual functionals differs dramatically for various properties and systems, and careful evaluation/calibration of the functional behavior must be performed before production DFT calculation can be used for interpreting any chemical problem.

Nice examples of such assessments are the calibration studies by Bühl and coworkers, ${ }^{8,12}$ which focused on the optimization of structures of transition-metal complexes, with DFT structures optimized in vacuo and referenced to structural parameters obtained in the gas phase. However, the geometries obtained in vacuo (or in an implicit solvent) are frequently inappropriately referenced to the structures determined by X-ray diffraction, neglecting any crystal effects. This approximation can lead to substantial errors and even to incorrect conclusions. ${ }^{13,14}$

To achieve the best available precision in the geometry optimizations, either molecular clusters based on experimental X-ray structures or periodic-boundary calculations should be used for calibrating the methods for solid-state structure optimization. In this work we focus on the cluster approach to assess the performance of the DFT functionals in optimizing the molecular geometry.

Recently, we applied this approach to a very limited number of complexes (mainly octahedral), ${ }^{10,15}$ but a wider selection of compounds and new functionals are required in order to draw more general conclusions. Therefore, the cluster-based comparison of ten DFT functionals (with or without D3 dispersion corrections) for structure optimization is presented in this work for seven square-planar complexes with various ligands and central metal atoms (Pt, Pd, Au, Rh, Fig. 1). These complexes were selected primarily based on the availability of high-quality $\mathrm{X}$-ray structures and the availability of complete NMR data and variability of central metals.

(ii) The solvent effects can be simulated using explicit solvent molecules or implicit solvent models. Whereas implicit solvent models in general are limited to accounting for
Pt 1

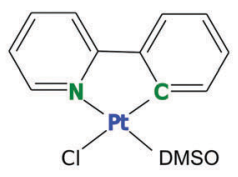

Pd 1

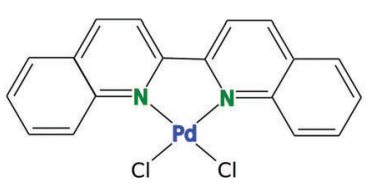

Au 1<smiles></smiles>
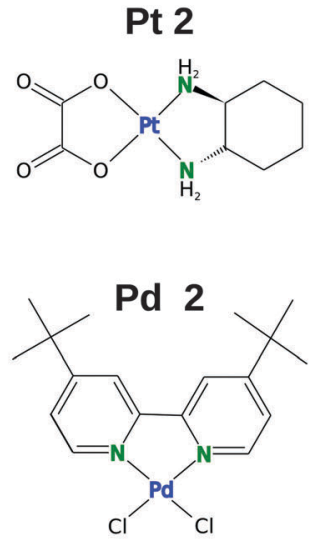

Au 2<smiles></smiles>

Rh

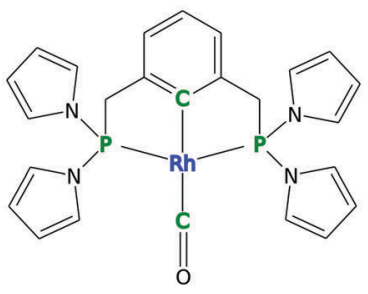

Fig. 1 Structures of selected square-planar complexes. The transitionmetal centers are shown in blue and the ligand spectator NMR atoms (LA) are shown in green.

electrostatic effects of the solvent environment (continuum) on the NMR parameters of the solute, ${ }^{16}$ an explicit solvent model accounts also for specific weak interactions (hydrogen bonding, stacking), ${ }^{17}$ which can significantly alter the NMR chemical shifts of atoms involved in these interactions. This applies in particular to the hydrogen atoms and the easily polarizable heavy element. ${ }^{18}$ The application of an explicit solvent model is, however, beyond the scope of everyday calculations due to its complexity. For a proper determination of the NMR chemical shifts, classical molecular dynamic (MD) or QM/MD simulations must be performed to determine the positions of the solvent molecules relative to the solute and to calculate NMR chemical shifts averaged over individual snapshots, which can be very time and resource consuming, although great advances are being made for NMR parameters of molecules containing light elements. ${ }^{19,20}$ Furthermore, computationally demanding QM/MD simulations are limited by the size of the model that can be evaluated in a reasonable period of time, whereas specialized force-field parameters for MD simulations must be developed for each structurally different molecule or non-standard solvent. ${ }^{21}$ Therefore, the explicit solvent model is used predominantly in detailed studies of individual systems, whereas the implicit solvent model is 
adopted more generally, being usually sufficient for calculations of many properties, including NMR chemical shifts of HALAinfluenced light atoms as we demonstrate in this work.

(iii) The relativistic effects can be treated in several ways at various levels of theory. A majority of the current computational codes operate with an approximate treatment of relativity. One of the most widely used approaches is the Zeroth-Order Regular Approximation. The ZORA Hamiltonian can be defined as: ${ }^{22}$

$$
\hat{H}^{\mathrm{ZORA}}=\hat{T}^{\mathrm{ZORA}}+\hat{V}=\boldsymbol{\sigma} \cdot \hat{\boldsymbol{p}} \frac{c^{2}}{2 m c^{2}-\hat{V}} \boldsymbol{\sigma} \cdot \hat{\boldsymbol{p}}+\hat{V}
$$

The relatively simple insertion of an electronic mean-field or the full Kohn-Sham potential into $\hat{T}^{\text {ZORA }}$ allows for the inclusion of SO contributions, yielding the two-component (2c) spin-orbit ZORA (SO-ZORA) approach. ${ }^{23,24}$

The SO-ZORA approach provides a good description of the valence-shell orbitals, often comparable with that of fourcomponent methods. ${ }^{23}$ Because the NMR chemical shifts of the light atoms $\left(\right.$ e.g. $\left.,{ }^{1} \mathrm{H},{ }^{13} \mathrm{C},{ }^{15} \mathrm{~N}\right)$ are significantly influenced by the type of chemical bonding, determined by the valence orbitals, the performance of the SO-ZORA approach for NMR chemical shift calculations of light atoms is good. ${ }^{4,7,25,26}$ However, it has been demonstrated ${ }^{10,11,15,27}$ that increasing the admixture of exact exchange in the functional is required to obtain correct NMR chemical shifts of atoms strongly affected by HALA effects. Recently, the role of the missing self-consistent first-order response of the DFT exchange-correlation (XC) potential to the external magnetic-field perturbation, the so-called XC response kernel $\left(f_{\mathrm{xc}}\right)$, has been highlighted in NMR chemical shift calculations using the SO-ZORA approach. ${ }^{28}$ The missing terms arising from $f_{\mathrm{xc}}$ were found to be quite sizable for hydrogen and mercury NMR chemical shifts. However, to the best of our knowledge, these effects have not been evaluated for light, non-hydrogen NMR atoms $\left({ }^{13} \mathrm{C},{ }^{15} \mathrm{~N}\right)$.

For a more complete inclusion of relativistic effects, the Dirac Hamiltonian must be considered: ${ }^{29}$

$$
\hat{H}^{\mathrm{D}}=c \boldsymbol{\alpha} \cdot \hat{\mathbf{p}}+\beta m c^{2}+\hat{V}
$$

In the Dirac formalism, operators and wave functions are represented as four-component objects. This will ultimately lead to more computationally demanding methods compared to approaches utilizing more approximate Hamiltonians. Until recently, such methods have been considered applicable only to relatively small molecular systems (up to 10-20 atoms), which greatly limited their general applicability to solve reallife chemical problems. However, modern four-component methods have now reached a level of maturity that make them very useful tools for modeling and understanding chemically interesting systems. ${ }^{30,31}$

Therefore, in addition to the two-component (2c) SO-ZORA approach, we employed four-component (4c) calculations in the Dirac-Coulomb framework. The fully relativistic NMR calculations (ReSpect program) use restricted magnetically balanced basis sets for the small-component wave function. ${ }^{32}$ To further improve basis-set convergence, gauge-including atomic orbitals (GIAO) are employed for the pure DFT functionals. ${ }^{33}$ In the case of the hybrid functionals, only the common gauge origin (CGO) methodology is implemented in the ReSpect program, and thus special attention must be paid to errors arising from basis-set incompleteness in these calculations. In contrast, the implementation of the state-of-the-art non-collinear DFT kernel for GGA (generalized gradient approximation) functionals is used. ${ }^{34}$

Here we evaluate the influence of structure, solvent, spinorbit contribution, exact-exchange admixture, role of $f_{\mathrm{xc}}$, and CGO approximation for hybrid functionals on relativistic NMR chemical shift calculations (2c and 4c) of light atoms in the vicinity of a heavy element for a series of square-planar transitional-metal complexes.

\section{Methods}

\subsection{Selection of complexes and experimental data}

A set of square-planar complexes with high-quality X-ray structures, complete NMR chemical shift data, and a diversity of the central metal atom was selected. The experimental data for the selected complexes are summarized in Table 1. In addition to "regular" square-planar platinum and palladium complexes with aromatic and aliphatic ligands (Pt and Pd), also complexes with ideal square-planar geometries distorted by the shape of the ligands, namely Au1 and Rh (see Fig. 1) are investigated. In Au1, the 2-benzoylpyridine ligand is bent out of the plane by $30^{\circ}$, whereas for $\mathbf{R h}$, where the square-planar shape is enforced

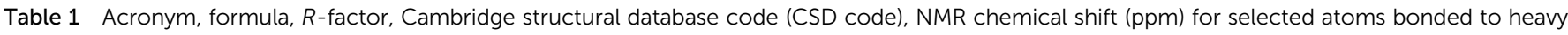

\begin{tabular}{|c|c|c|c|c|c|c|}
\hline Acronym & Formula & $R$-factor (\%) & CSD code & NMR chemical shift ${ }^{a}$ & NMR solvent & Ref. \\
\hline Pt1 & $\operatorname{trans}(S, N)-\left[\mathrm{Pt}\left(2-\mathrm{ppy}^{*}\right)(\mathrm{DMSO}) \mathrm{Cl}\right]$ & 1.8 & JISPAD01 & $\begin{array}{l}{ }^{15} \mathrm{~N} 220.6 \\
{ }^{13} \mathrm{C} 140.2\end{array}$ & DMSO-d 6 & 35 and 36 \\
\hline Pt2 & {$[\mathrm{Pt}($ oxalato $)(1 R, 2 R$-cyclohexanediamine $)]$} & 1.9 & CUHKEV & ${ }^{15} \mathrm{~N} 9.1^{b}$ & DMSO- $_{6}$ & 37 \\
\hline Pd1 & {$\left[\mathrm{Pd}\left(2,2^{\prime}\right.\right.$-biquinoline $\left.) \mathrm{Cl}_{2}\right]$} & 2.7 & YASPAK & ${ }^{15} \mathrm{~N} 224.0$ & $\mathrm{CDCl}_{3}$ & 38 and 39 \\
\hline Pd2 & {$\left[\operatorname{Pd}(4,4\right.$-di-tert-butylbipyridine $\left.) \mathrm{Cl}_{2}\right]$} & 4.8 & MOYWIG01 & ${ }^{15} \mathrm{~N} 211.1$ & DMSO-d 6 & 40 and 41 \\
\hline Au1 & [Au(2-benzoylpyridine $\left.) \mathrm{Cl}_{2}\right]$ & 2.9 & PUKYAV & $\begin{array}{l}{ }^{15} \mathrm{~N} 215.2 \\
{ }^{13} \mathrm{C} 136.7\end{array}$ & $\mathrm{DMSO}^{-\mathrm{d}_{6}}$ & 42 and 43 \\
\hline Au2 & [Au(2-phenylpyridine) $\left.\mathrm{Cl}_{3}\right]$ & 2.2 & YIDMAA & ${ }^{15} \mathrm{~N} 227.7$ & $\mathrm{CDCl}_{3}$ & 44 and 45 \\
\hline $\mathbf{R h}$ & [Rh(dipyrrolylphoshinoxylene) CO] & 3.3 & SOWDUE & $\begin{array}{l}{ }^{31} \mathrm{P} 119.8 \\
{ }^{13} \mathrm{C} 172.2\end{array}$ & THF- $\mathrm{d}_{8}$ & 46 \\
\hline
\end{tabular}
metal (highlighted in Fig. 1), NMR solvent used in the study, and corresponding references for selected square-planar complexes

${ }^{a}$ All ${ }^{15} \mathrm{~N}$ NMR chemical shifts are reported relative to liquid $\mathrm{NH}_{3} \cdot{ }^{b}$ This work. 
by the so-called PCP "pincer" ligand, the bond angles around the central metal are about $12^{\circ}$ distorted from the ideal $90^{\circ}$.

\subsection{Geometry optimization - DFT functional and basis set}

Molecular cluster models were prepared for each complex individually, with a single central molecule (color-coded in Fig. 2) completely surrounded by crystal-packing molecules (orangecoded in Fig. 2) in a way that all close-contact interactions between the central molecule and the packing ones were treated in the final cluster. As a result, the central molecule was completely surrounded in a crystal-like environment with a packing sphere up to $12 \AA$, depending on the size of the central and packing molecules. Subsequently, the coordinates of the packing molecules were fixed, whereas the geometry of the central molecule was optimized using different DFT functionals in the field created by packing ones. This approach is assumed to recover the majority of the crystal lattice effects ${ }^{10,15,47-50}$ allowing for a direct comparison of calculated parameters to experimental X-ray data. An example of the molecular cluster for Au1 is shown in Fig. 2.

The geometries of the central molecules in the clusters were optimized using the def2-TZVPP basis set ${ }^{51}$ for all atoms, with corresponding relativistic effective core potentials (def2ECPs) ${ }^{52}$ for the metal center (ECP substituting 60 electrons for $\mathrm{Pt}$ and $\mathrm{Au}$ and 28 electrons for $\mathrm{Rh}$ and $\mathrm{Pd}$ ). The packing molecules with fixed coordinates were treated using the def2SVP basis set ${ }^{51}$ for all atoms, with corresponding ECP for the metal center.

Each cluster was optimized using ten selected density functionals. The LYP-based ${ }^{53}$ functionals BLYP, ${ }^{54}$ B3LYP, ${ }^{55}$ and BHLYP $^{56}$ were selected to map the effect of exact-exchange admixture on the structural parameters. Due to the known unbalanced performance of the B3LYP functional in calculations involving transition metals, ${ }^{57}$ the CAM-B3LYP, ${ }^{58}$ the B3LYP functional with corrected long-range exchange, was included in the test set. From the GGA family, $\mathrm{PBE}^{59}$ and $\mathrm{BP}^{5} 6^{54,60}$ are

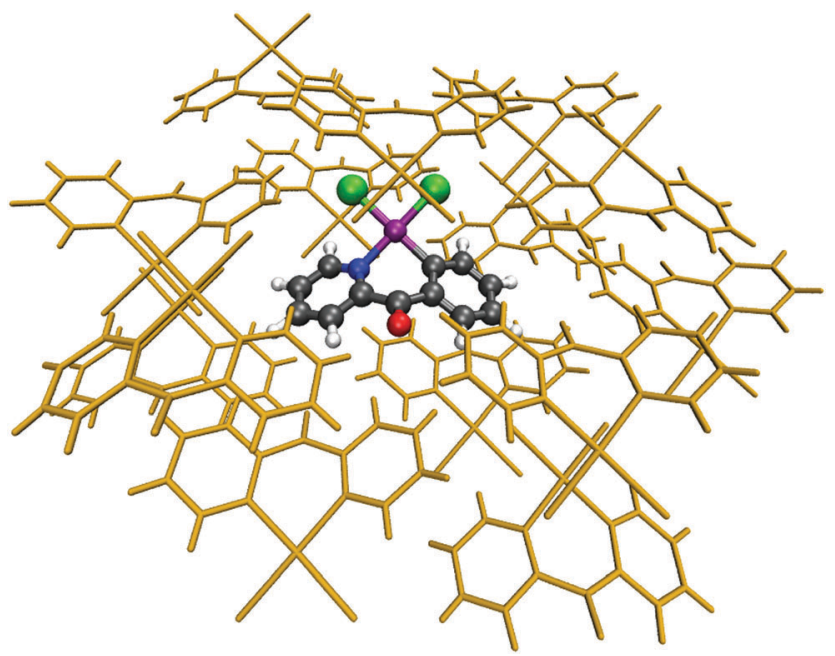

Fig. 2 Molecular cluster of Au1 with central (in color) and packing (in orange) molecules. present because they are known to perform reasonably well for transition-metal complexes. ${ }^{12}$ The TPSSH $^{61,62}$ functional is an example of a successful meta-GGA hybrid functional, and it was shown to be a very good choice for optimizing transitionmetal complexes. ${ }^{8,12}$ The PBE0, ${ }^{63,64}$ the hybrid version of the "parameter-free" PBE functional, has demonstrated superb performance for geometry optimization of heavy transitionmetal compounds. ${ }^{10,12,15}$ Advanced long-range corrected hybrid functional with empirical dispersion corrections, $\omega \mathrm{B} 97 \mathrm{XD},{ }^{65,66}$ was recently tested with good results in structure optimizations of transition-metal complexes. ${ }^{67}$ Furthermore, a recent addition to the family of "Minnesota functionals", the so-called screened-exchange density functional, MN12SX, ${ }^{68}$ which was reported to offer very good performance in calculating various properties, ${ }^{68}$ is included. The effect of the D3 dispersion correction $^{69}$ on the geometry was tested in several cases, see Fig. S1 (ESI $\dagger$ ). The observed effect was found negligible for the PBE0 functional (it even deteriorates the results by $0.2 \mathrm{pm}$ for PBE and BP86), and the D3 correction is thus not further considered in this work.

A convergence of the basis set was estimated in the series def2-SVP $\rightarrow$ def2-QZVPP using the PBE0 functional, see Fig. 3. The def2-TZVPP basis provides results almost identical (difference $<1 \mathrm{pm}$ ) to those of much larger (and computationally demanding) QZ bases, confirming our previous results. ${ }^{10}$

Based on the performance of individual functionals (vide infra), the basis-set effects (Fig. 3), computational costs, and the marginal effects of dispersion correction, the PBE0/def2-TZVPP/ ECP approach was used to optimize molecular geometries in the production calculations. For all the in-solution optimizations discussed below, the COSMO (COnductor-like Screening MOdel) ${ }^{16}$ solvent model was used, see Section 2.3.

\subsection{NMR chemical shift calculations - solvent effects and method selection}

Geometry. To calculate in-solution NMR chemical shifts, individual single molecules were optimized using the PBE0 with the def2-TZVPP basis set for all atoms and corresponding ECP for the transition metal (the approach calibrated in Section 2.2

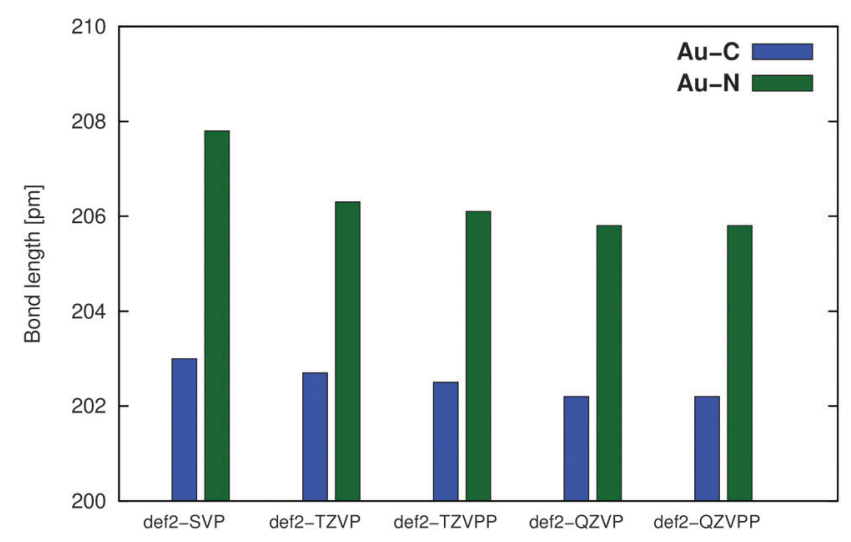

Fig. 3 Basis-set effects on the $M-L$ distance in the optimization of compound Au1 using the PBEO functional with def2-ECP. 
with respect to the experimental X-ray reference geometries). In addition, the implicit COSMO solvent model ${ }^{16}$ was adopted throughout the following geometry optimizations. The standard COSMO parameters as implemented in the ADF program (DMSO - the relative dielectric constant $\varepsilon=46.7$, the radius of the solvent probe $r_{\text {solv }}=3.04 \AA ; \mathrm{CHCl}_{3}-\varepsilon=4.8, r_{\text {solv }}=3.17 \AA$; $\mathrm{THF}-\varepsilon=7.6, r_{\text {solv }}=3.18 \AA$; benzene $-\varepsilon=2.3, r_{\text {solv }}=3.28 \AA$ ) were used.

Two-component (2c) relativistic NMR calculations. Twocomponent (2c) relativistic NMR calculations were performed using the SO-ZORA approach (as implemented in the ADF package $)^{24,70,71}$ and PBE, PBE0, or PBE0 with an exact-exchange admixture of $40 \%$ (PBE-40). The choice of the functional is justified by our previous studies ${ }^{10,11}$ and the dependence on the basis set was tested in this study (Fig. 4). As the difference between the NMR chemical shifts $\left({ }^{13} \mathrm{C}\right.$ and $\left.{ }^{15} \mathrm{~N}\right)$ for Au1 calculated using TZP and QZ4P basis sets is $\sim 1 \mathrm{ppm}$ (Fig. 4), the standard TZP basis set from the ADF library was used for all atoms in the production calculations. All calculations were performed in vacuo as well as using the COSMO solvent model in order to estimate the effect of the solvent on the NMR chemical shifts. The gauge-origin dependence was handled using gaugeincluding atomic orbitals (GIAOs). ${ }^{72}$

Four-component (4c) relativistic NMR calculations. Fourcomponent (4c) relativistic NMR calculations were performed using the Dirac-Coulomb Hamiltonian (as implemented in the ReSpect code $)^{32,33,73}$ using PBE, PBE0, and PBE-40 functionals, with the uncontracted pc-2 basis set ${ }^{74,75}$ for the light atoms and the uncontracted Dyall's valence triple-zeta (dyall-vtz) basis set $^{76-78}$ for the central metal. All shielding calculations using the ReSpect code were performed in vacuo; the solvent corrections were estimated at the SO-ZORA level as $\left(\sigma_{\text {COSMO }}-\sigma_{\text {vacuo }}\right)$ and added to the NMR chemical shifts calculated at the fourcomponent mDKS level. The PBE calculations were performed using GIAOs to treat the gauge-origin dependence whereas for the PBE0 and PBE-40 functionals, the Common Gauge Origin (CGO) approximation, with the GO placed on the metal center, was used. Because the CGO and GIAO calculations are directly
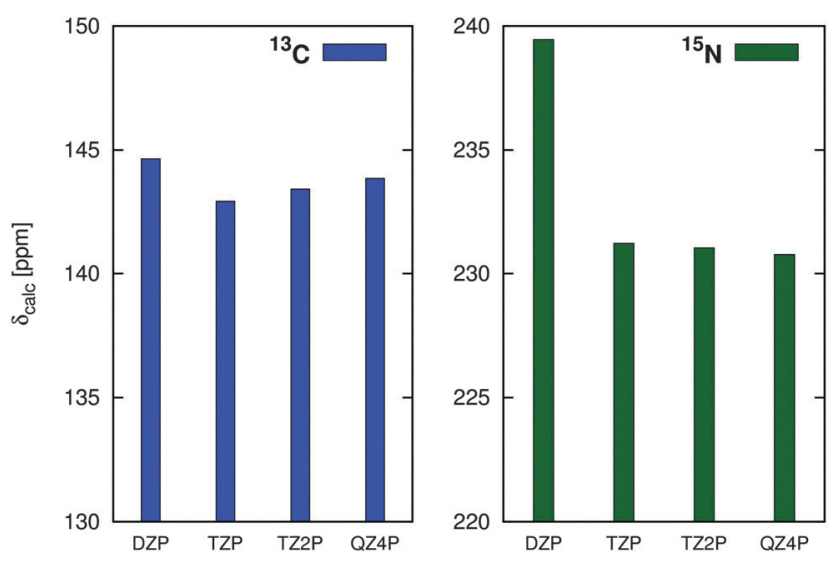

Fig. 4 Basis set effects on the ${ }^{13} \mathrm{C}$ and ${ }^{15} \mathrm{~N}$ NMR chemical shifts in compound Au1 calculated at the SO-ZORA level using the PBE-40 functional (PBE0/def2-TZVPP/def2-ECP geometry). compared, we used empirical CGO $\rightarrow$ GIAO correction to account for the basis set incompleteness error in the CGO calculations. This correction is obtained for each atom as a difference between shielding calculated using GIAO and CGO approximations at the PBE level $\left(\sigma_{\mathrm{PBE}(\mathrm{CGO})}-\sigma_{\mathrm{PBE}(\mathrm{GIAO})}\right)$ and was added to the shielding constant calculated at the PBE0 or PBE-40 level. Inclusion of the "CGO correction" significantly improves the agreement with experiment (see Section 3.2 and ESI $\dagger$ ).

Effect of the kernel of the exchange-correlation functional $\left(f_{\mathrm{xc}}\right)$ on the NMR chemical shifts. Non-vanishing two-electron contributions $\left(f_{2 \mathrm{e}}\right)$ to the NMR shielding response equations consist of the exchange-correlation $\left(f_{\mathrm{xc}}\right)$ and exact-exchange $\left(f_{\mathrm{ex}}\right)$ kernels $\left(f_{2 \mathrm{e}}=f_{\mathrm{xc}}+f_{\mathrm{ex}}\right)$. In the absence of spin-orbit (SO) effects, there is no contribution from the kernel of the exchange-correlation functional $\left(f_{\mathrm{xc}}\right)$ to the NMR shielding calculations. Thus in the framework of pure DFT, NMR shielding constants can be calculated in non-relativistic or scalarrelativistic theories using simple sum-over-states expressions (note that in the Hartree-Fock or hybrid DFT framework, the kernel arising from the exact exchange $\left(f_{\text {ex }}\right)$ cannot be neglected at any relativistic or non-relativistic level). However, in the presence of the SO coupling, the contribution from the exchange-correlation kernel $\left(f_{\mathrm{xc}}\right)$ is non-vanishing and can give significant contributions to the NMR shielding constants, especially for the light atom (LA) directly bonded to the heavy-atom center. For example, in the case of hydrogen iodide, the exchange-correlation kernel contributes more than $3 \mathrm{ppm}$ to the total ${ }^{1} \mathrm{H}$ NMR shielding constant. ${ }^{32}$ Because this effect is missing from the calculations of standard reference compounds (e.g., tetramethylsilane for ${ }^{13} \mathrm{C}$, ammonia for ${ }^{15} \mathrm{~N}$ ) it fully manifests itself in the relativistic NMR chemical shift calculations.

To determine the importance of $f_{\mathrm{xc}}, 4 \mathrm{c}$ calculations using the ReSpect code were performed also without $f_{\mathrm{xc}}$, and the corresponding correction to the NMR chemical shift, $\Delta \delta_{\mathrm{kernel}}^{\mathrm{XC}}$, was obtained as a difference between the NMR chemical shift calculated with and without kernel contribution at a given level of theory. With $\Delta \delta_{\text {kernel }}^{\mathrm{XC}}$ being unaffected by the gauge origin (GIAO vs. CGO), (tested using GIAO/CGO calculations at the 4c-PBE level, data not shown) we assume its additivity for the $2 \mathrm{c}$ SO-ZORA results (vide infra).

NMR chemical shift referencing. To reduce systematic errors in calculating the NMR chemical shifts, the following secondary references were used. For ${ }^{15} \mathrm{~N}, \mathrm{~N}$-methylpyridinium in DMSO ( $\delta=203.5 \mathrm{ppm}$ relative to liquid ammonia) $;^{79,80}$ for ${ }^{13} \mathrm{C}$, benzene in benzene ( $\delta=127.83 \mathrm{ppm}$ relative to TMS $) ;{ }^{4}$ and for ${ }^{31} \mathrm{P}$, tert-butylphosphine in $\mathrm{CHCl}_{3}(\delta=63.0 \mathrm{ppm}$ relative to $\left.85 \% \mathrm{H}_{3} \mathrm{PO}_{4}\right) .{ }^{81}$ The NMR chemical shifts, $\delta_{\mathrm{i}}$, were obtained using the equation:

$$
\delta_{\mathrm{i}}=\sigma_{\text {ref }}-\sigma_{\mathrm{i}}+\delta_{\text {ref }}
$$

where $\sigma_{\text {ref }}$ is the calculated NMR shielding of the corresponding nucleus in the reference compound, $\sigma_{\mathrm{i}}$ is the calculated NMR shielding of the investigated nucleus, and $\delta_{\text {ref }}$ is the experimental NMR chemical shift of the secondary reference relative to the primary standard. 


\section{Results and discussion}

3.1 Geometry optimization - estimating the structural effects on the NMR chemical shift

The molecular clusters were built based on X-ray diffraction geometries (for details, see Methods Section) and the central molecule of each cluster was optimized in the field created by fixed packing molecules. The performance of individual functionals was evaluated using bond distances for non-hydrogen atoms (comparison with the experimental values for 161 bonds) with special focus on 28 bonds between the central metal and the ligand atoms. Root-mean-square deviations (RMSD) between calculated and experimental values for all 161 non-hydrogen bonds $\left(\mathrm{RMSD}_{161}\right)$ and for 28 bonds around the metal center $\left(\mathrm{RMSD}_{28}\right)$ from all molecules of our set are summarized in Fig. 5.

Poor results are obtained by using the BLYP functional, which greatly overestimates the $\mathrm{M}-\mathrm{L}$ bonds $\left(\mathrm{RMSD}_{28}=5.2 \mathrm{pm}\right)$ and thus should not be used for structure optimizations of transition-metal complexes. ${ }^{10}$ Increasing the exact-exchange admixture in the LYPbased functionals improves the correlation with experimental data for M-L bonds [BLYP $(0 \%, 5.2 \mathrm{pm}) \rightarrow$ B3LYP $(20 \%, 3.3 \mathrm{pm}) \rightarrow$ $\operatorname{BHLYP}(50 \%, 2.4 \mathrm{pm})]$ notably. When all bond lengths are considered, both B3LYP and BHLYP are performing with the same level of accuracy $\left(\mathrm{RMSD}_{161} \sim 2 \mathrm{pm}\right)$, which is due to the fact that the impact of non-metal ligand bonds (mostly organics, in which B3LYP is known to perform excellently) compensates for the inaccuracy in describing the $\mathrm{M}-\mathrm{L}$ bonds. The best of the LYP-based functionals, BHLYP, produced moderately accurate results $\left(\mathrm{RMSD}_{28}=2.4 \mathrm{pm}, \mathrm{RMSD}_{161}=1.9 \mathrm{pm}\right)$ compared to the rest of the functionals. However, almost identical performance was observed for PBE and BP86, at notably lower computational costs. Both of these GGA functionals can be recommended for geometry optimization of transition-metal complexes in cases where the use of hybrid GGAs is inconvenient, e.g. for very large molecules or molecular clusters.

The $\omega \mathrm{B} 97 \mathrm{XD}$ functional produces good overall results $\left(\mathrm{RMSD}_{161}=1.7 \mathrm{pm}\right)$, whereas the description of the M-L bonding is comparable to BHLYP, BP86, and PBE.

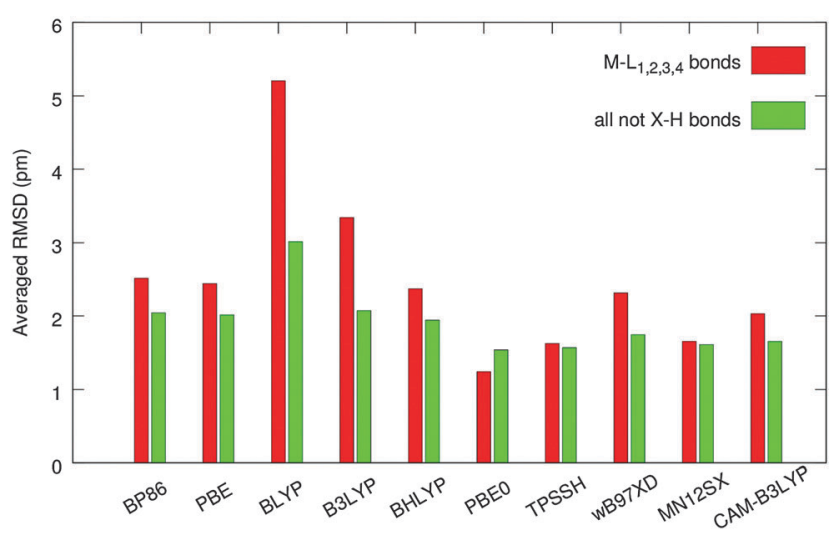

Fig. 5 The averaged RMSDs (in pm) for interatomic distances relative to the experimental $X$-ray data, calculated using various density functionals. The $M-L$ bonds (28) are in red, and all non-hydrogen bonds (161) are in green.
The importance of corrected long-range exchange in the CAM-B3LYP functional to optimize the geometry of the transitionmetal complexes is obvious from a comparison of the B3LYP and CAM-B3LYP results. The CAM version improves the results of B3LYP by $40 \%$ for M-L bonds (RMSD 28 is $2.0 \mathrm{pm} v s .3 .3 \mathrm{pm}$ for B3LYP) and by about $20 \%$ for all non-hydrogen bonds (RMSD $_{161}$ is 1.7 pm vs. 2.1), see Fig. 5.

The best results for the tested set were produced by three functionals - MN12SX $\left(\mathrm{RMSD}_{28}=1.7 \mathrm{pm}\right.$ and $\left.\mathrm{RMSD}_{161}=1.6 \mathrm{pm}\right)$, TPSSH (with $\mathrm{RMSD}_{28}=\mathrm{RMSD}_{161}=1.6 \mathrm{pm}$ ), and PBE0, which performs excellently in describing the $\mathrm{M}-\mathrm{L}$ bonds $\left(\mathrm{RMSD}_{28}=\right.$ $\left.1.2 \mathrm{pm}, \mathrm{RMSD}_{161}=1.5 \mathrm{pm}\right)$. For a more detailed analysis of the results according to the atom type (Fig. S2 and S3, ESI $\dagger$ ) and for a comparison between the cluster approach and the in vacuo results (Fig. S4), see ESI. $\dagger$

To estimate the magnitude of the structural effects on the NMR chemical shifts (NMR CS) separately from all the other factors, we calculated the NMR CS of metal-bonded light atoms (highlighted in green in Fig. 1) using non-relativistic and SO-ZORA approaches at the PBE-40/TZP level in vacuo for BLYP, B3LYP, PBE0, and MN12SX geometries (def2-TZVPP/def2-ECP basis set) optimized in vacuo (see Table 2). The difference between the NMR CS calculated using BLYP and MN12SX functionals $\left(\Delta \delta_{\text {geom }}\right.$, see Table 2$)$ ranges between 5-14 ppm at the non-relativistic level, and 2-22 ppm at the 2c SO-ZORA level. Also, the deficiency of the popular B3LYP functional in describing the $\mathrm{M}-\mathrm{L}$ bond length can result in a $19 \mathrm{ppm}$ difference in the relativistic ${ }^{13} \mathrm{C}$ NMR chemical shift $\left(e . g .,{ }^{13} \mathrm{C}\right.$ in $\mathbf{A u 1}$ calculated at the 2c SO-ZORA level). We conclude that the inaccurate geometry can be reflected in the ${ }^{13} \mathrm{C}$ and ${ }^{15} \mathrm{~N}$ NMR CS deviations, amounting easily to as much as $10-20 \mathrm{ppm}$. These deviations are more pronounced for the NMR chemical shifts calculated at the relativistic level (SO-ZORA) compared to those calculated using the non-relativistic approach (Table 2). This relativistic effect is particularly evident for the ${ }^{13} \mathrm{C}$ NMR CS in Au1 due to the more covalent character of the $\mathrm{M}-\mathrm{C}$ bond compared to that of the $\mathrm{M}-\mathrm{N}$ bond (Table 2). ${ }^{11,82}$ As expected, the PBE0, TPSSH, and MN12SX functionals, producing the most reliable geometries in the test set, give very similar calculated NMR CS.

In this work, the PBE0/def2-TZVPP/def2-ECP approach was selected for all further geometry optimizations in Sections 3.2-3.5.

3.2 Relativistic calculations of NMR chemical shifts (NMR CS) role of exact-exchange (EE) admixture

All the NMR chemical shifts were calculated for single molecules optimized using the PBE0 functional (for details, see Methods Section and Section 3.1), however, only those that require relativistic treatment, namely atoms significantly affected by the HALA effects from the metal atom (see Fig. 1 and Introduction part), are reported here for clarity. It is known that the calculated NMR CS are significantly modulated by the amount of exact-exchange admixture in the hybrid GGA functional. ${ }^{11,27}$ To analyze the role of exact-exchange admixture, we performed relativistic calculations of the light-atom NMR CS using 2c SO-ZORA and 4c mDKS with the PBE, PBE0, 
Table 2 NMR chemical shifts calculated at non-relativistic and relativistic (SO-ZORA) levels of theory using PBE-40/TZP in vacuo for compounds Pt1 and Au1 optimized using BLYP, B3LYP, PBE0, or MN12SX functionals (def2-TZVPP/def2-ECP). NMR chemical shifts in ppm (for shift referencing, see Methods Section)

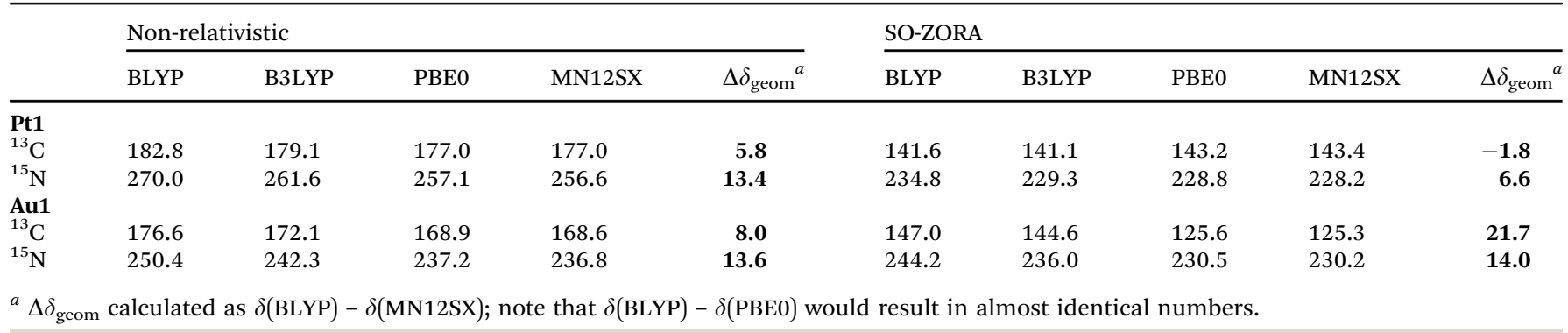

and PBE-40 functionals. Results (in vacuo), plotted as deviations from the experimental values $(\Delta \delta)$, are shown in Fig. 6 and more details are given in Tables S1 and S2 (ESI $\dagger$ ); for the influence of the CGO correction in the 4c mDKS PBE0 and PBE-40 calculations, see Fig. S5 (ESI $\dagger$ ).

Generally, increasing the EE admixture in the functional increases the nuclear magnetic shielding of the light atoms bound to the metal center in platinum and gold compounds considerably. ${ }^{10,82}$ However, this effect is marginal for palladium and even reversed for ${ }^{31} \mathrm{P}$ in the rhodium complex. The increased EE admixture improves the results for some cases already in vacuo, particularly for ${ }^{13} \mathrm{C}$ NMR CS, where the solvent effects obviously play a less significant role compared to ${ }^{15} \mathrm{~N}$ NMR CS. ${ }^{80,83-85}$ For instance, the PBE functional results in $\Delta \delta\left({ }^{13} \mathrm{C}\right)=$ $21.4 \mathrm{ppm}$ for Pt1-C, whereas PBE-40 gives $\Delta \delta\left({ }^{13} \mathrm{C}\right)=1.5 \mathrm{ppm}$ (see Fig. 6). Conversely, the improvement is small or negligible for ${ }^{15} \mathrm{~N}$ NMR CS, where solvent effects are known to play a significant role and the effects of the EE admixture are less important (see the palladium and gold complexes in Fig. 6).

\subsection{Solvent correction to the NMR CS}

Due to the sensitivity of the NMR CS (particularly ${ }^{15} \mathrm{~N}$ ) ${ }^{85}$ to the solvent, it is necessary to include bulk environmental effects in the NMR calculations. ${ }^{86}$ In this study, we analyzed the effect of the implicit solvent model on the NMR CS of LA $\left({ }^{13} \mathrm{C}\right.$ and $\left.{ }^{15} \mathrm{~N}\right)$. Solvent corrections to the NMR CS $\left(\Delta \delta_{\text {solv }}\right)$, calculated at the SO-ZORA level using the PBE, PBE0, and PBE-40 functionals with the COSMO model for the solvent, are summarized in Table 3.

The implicit solvent improved the $\mathrm{RMSD}_{\mathrm{PBE}}^{\mathrm{SO}-\mathrm{ZORA}}$ from $21.5 \mathrm{ppm}$ in vacuo to $14 \mathrm{ppm}$ with COSMO (for $\mathrm{RMSD}_{\mathrm{PBE}}^{\mathrm{mDKS}}$, $18.6 \mathrm{ppm}$ in vacuo and $11.4 \mathrm{ppm}$ including $\Delta \delta_{\text {solv }}$ ). In parallel, the RMSD ${ }_{\mathrm{PBEO}}^{\mathrm{SO}-Z \mathrm{R} A}$ was reduced from $16.5 \mathrm{ppm}$ to $4.4 \mathrm{ppm}$ $\left(\mathrm{RMSD}_{\mathrm{PBEO}}^{\mathrm{mDKS}}\right.$ from $17.5 \mathrm{ppm}$ to 5.1$)$ and the RMSD ${ }_{\mathrm{PBE}-40}^{\mathrm{SO}-\mathrm{ZORA}}$ from $14.6 \mathrm{ppm}$ to $4.5 \mathrm{ppm}$ (RMSD $\mathrm{RBEE}_{\mathrm{PBO}}^{\mathrm{mDKS}} 15.1 \mathrm{ppm}$ to $5.2 \mathrm{ppm}$ ).

Note that the magnitude of $\Delta \delta_{\text {solv }}$ is increasing considerably with increasing exact-exchange admixture in the functional; the more exact exchange in the functional, the more important the solvent correction is, which is partially reflected in the decrease of the RSMD for the individual approaches. Whereas PBE results for both the 2c SO-ZORA and 4c mDKS approaches still deviate significantly from the experimental values, which is partially due to the differences in the description of $\Delta \delta_{\text {so }}$ by the individual functionals (see Table 4), PBE0 and PBE-40 results are considerably closer to the experimental data. For the differences between calculated (PBE, PBE0, or PBE-40 functional using implicit solvent) and experimental NMR CS, see Fig. 7.

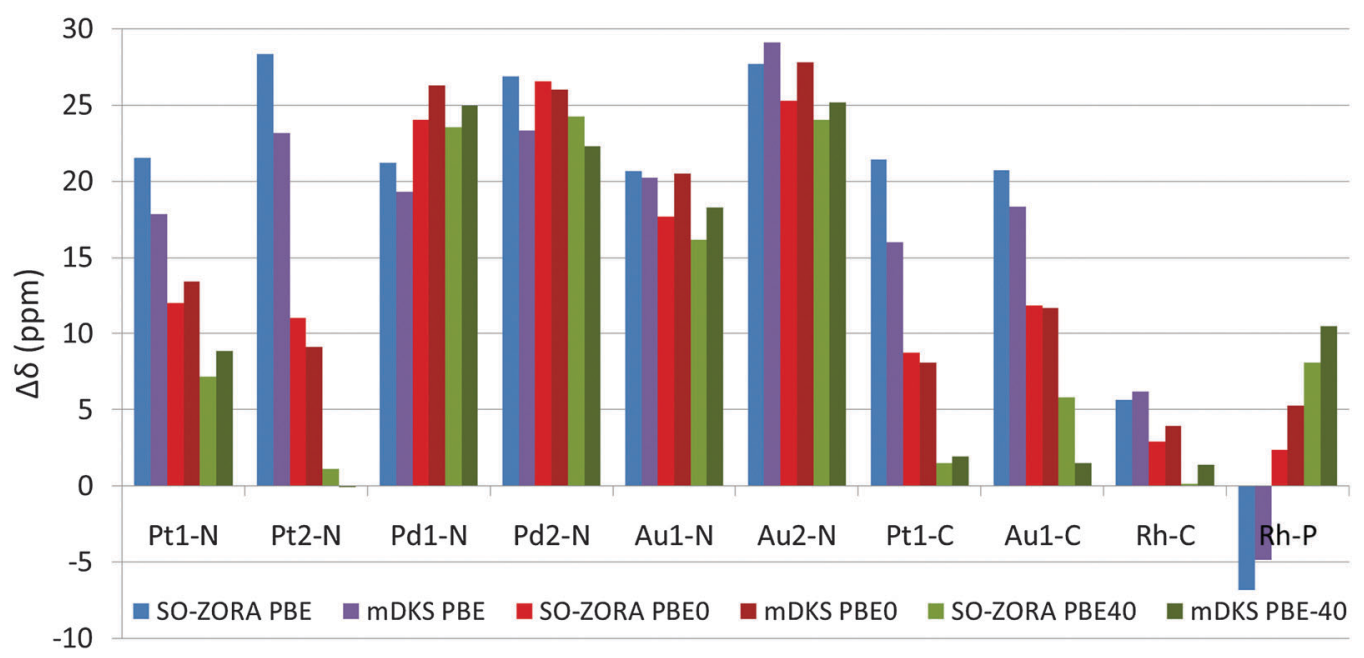

Fig. 6 Total NMR chemical shifts calculated using 2c SO-ZORA and 4c mDKS methods at various levels of theory in vacuo (for details, see Methods section). The calculated values are reported as deviations $(\Delta \delta)$ from the experimental NMR chemical shifts. 
Table 3 The $\Delta \delta_{\text {solv }}$ calculated at PBE, PBEO, and PBE-40 levels of theory using the 2c SO-ZORA method

\begin{tabular}{lrrr}
\hline$\Delta \delta_{\text {solv }}$ & & & \\
\hline Atom & PBE & PBE0 & PBE-40 \\
\hline Pt1-N & -5.0 & -5.7 & -6.1 \\
Pt2-N & -1.3 & -4.4 & -6.3 \\
Pd1-N & -4.6 & -14.6 & -20.4 \\
Pd2-N & -6.0 & -16.8 & -22.2 \\
Au1-N & -12.3 & -15.7 & -17.5 \\
Au2-N & -14.5 & -17.9 & -20.1 \\
Pt1-C & -4.1 & -4.4 & -4.5 \\
Au1-C & -2.5 & -3.4 & -4.1 \\
Rh-C & -0.2 & -0.3 & -0.2 \\
Rh-P & +2.7 & +2.2 & +2.0 \\
& & &
\end{tabular}

Although at the SO-ZORA relativistic level with solvent correction, the PBE0 and PBE-40 calculations result in almost identical averaged RMSD values $\left(\mathrm{RMSD}_{\mathrm{PBEO}}^{\mathrm{SO}-\mathrm{ZORA}}=4.4 \mathrm{ppm}\right.$ and $\mathrm{RMSD}_{\mathrm{PBE}-40}^{\mathrm{SO}}=4.5 \mathrm{ppm}$ ), the PBE-40 provides better results in 7 out of 10 systems, which is in agreement with previous studies. ${ }^{10,11}$ The better average RMSD achieved by the PBE0 functional can be ascribed to quite poor results of the PBE-40 approach for Pt2- $\mathrm{N}, \mathrm{NH}_{2}$ group in oxaliplatin, which is probably involved in protonation equilibria affecting its experimental ${ }^{15} \mathrm{~N}$ NMR CS, and overestimated ${ }^{31} \mathrm{P}$ NMR chemical shift for Rh-P. When the two abovementioned problematic Pt2-N and Rh-P cases are excluded, $\mathrm{RMSD}_{\mathrm{PBE}-40}^{\mathrm{SO}}$ decreases to $2.4 \mathrm{ppm}$, whereas $\mathrm{RMSD}_{\mathrm{PBEO}}^{\mathrm{SO}-\mathrm{ZORA}}=4.7 \mathrm{ppm}$.

Similar results were achieved by the $4 \mathrm{c}$ mDKS approach, where slightly better RMSD was obtained by using PBE0 $\left(\mathrm{RMSD}_{\mathrm{PBE}}^{\mathrm{mDKS}}=5.1 \mathrm{ppm}\right)$ than by PBE-40 $\left(\mathrm{RMSD}_{\mathrm{PBE}-40}^{\mathrm{mDKS}}=5.2 \mathrm{ppm}\right)$, however, one should keep in mind that this was achieved by applying several external corrections (solvent, CGO).

Given the good results obtained for PBE0 and PBE-40 with an implicit solvent correction, the use of explicit solvent models would not bring any considerable improvement at reasonable costs for routine NMR applications; therefore, we use the COSMO implicit solvent model for calculating the NMR CS in subsequent sections.

\subsection{Spin-orbit contribution to the NMR CS}

It is well known that most of the HA-LA effect is due to spinorbit coupling. ${ }^{86-89}$ Hence it is vitally important to observe and analyze the difference between calculations with and without the SO contribution $\left(\Delta \delta_{\mathrm{SO}}\right)$, i.e., the SO in ZORA as well as the

Table 4 The $\Delta \delta_{\text {So }}$ calculated at SO-ZORA and mDKS levels of theory using PBE, PBE0, and PBE-40 functionals in vacuo

\begin{tabular}{|c|c|c|c|c|c|c|c|c|c|c|}
\hline \multirow[b]{2}{*}{ Atom } & \multicolumn{5}{|c|}{$\underline{\Delta \delta_{\mathrm{SO}}}(\mathrm{ZORA})$} & \multicolumn{5}{|c|}{$\Delta \delta_{\mathrm{SO}}(\mathrm{mDKS})$} \\
\hline & PBE & PBE0 & PBE-40 & $\Delta \Delta \delta_{\mathrm{SO}}{ }^{a}$ & $\Delta \Delta \delta_{\text {total }}^{b}$ & PBE & PBE0 & PBE-40 & $\Delta \Delta \delta_{\mathrm{SO}}^{a}$ & $\Delta \Delta \delta_{\text {total }}^{b}$ \\
\hline Pt1-N & -9.3 & -15.2 & -19.1 & -9.8 & -14.4 & -10.7 & -15.7 & -19.0 & -8.3 & -9.0 \\
\hline Pd1-N & -5.1 & -9.3 & -12.4 & -7.3 & +2.4 & -5.6 & -9.5 & -12.6 & -7.0 & +5.7 \\
\hline Pd2-N & -7.1 & -11.5 & -15.1 & -8.0 & -2.7 & -8.6 & -13.8 & -17.6 & -9.0 & -1.0 \\
\hline Au1-N & -2.7 & -3.5 & -4.5 & -1.8 & -4.6 & 0.6 & -1.5 & -3.0 & -3.6 & -1.9 \\
\hline Au2-N & 6.0 & 5.6 & 5.0 & -1.0 & -3.6 & 9.9 & 7.3 & 6.3 & -3.6 & -3.9 \\
\hline Rh-C & -3.1 & -4.3 & -5.9 & -2.8 & -5.5 & -2.5 & -4.5 & -5.8 & -3.3 & -4.8 \\
\hline Rh-P & -17.0 & -22.0 & -24.9 & -7.9 & +14.9 & -5.1 & -10.3 & -12.7 & -7.6 & +15.3 \\
\hline
\end{tabular}

${ }^{a}$ Calculated as $\Delta \Delta \delta_{\mathrm{SO}}=\Delta \delta_{\mathrm{PBE}-40}^{\mathrm{SO}}-\Delta \delta_{\mathrm{PBE} \cdot{ }^{b}}^{\mathrm{SO}}$ Calculated as $\Delta \Delta \delta_{\text {total }}=\Delta \delta_{\mathrm{PBE}-40}^{\text {total }}-\Delta \delta_{\mathrm{PBE}}^{\text {total }}$.

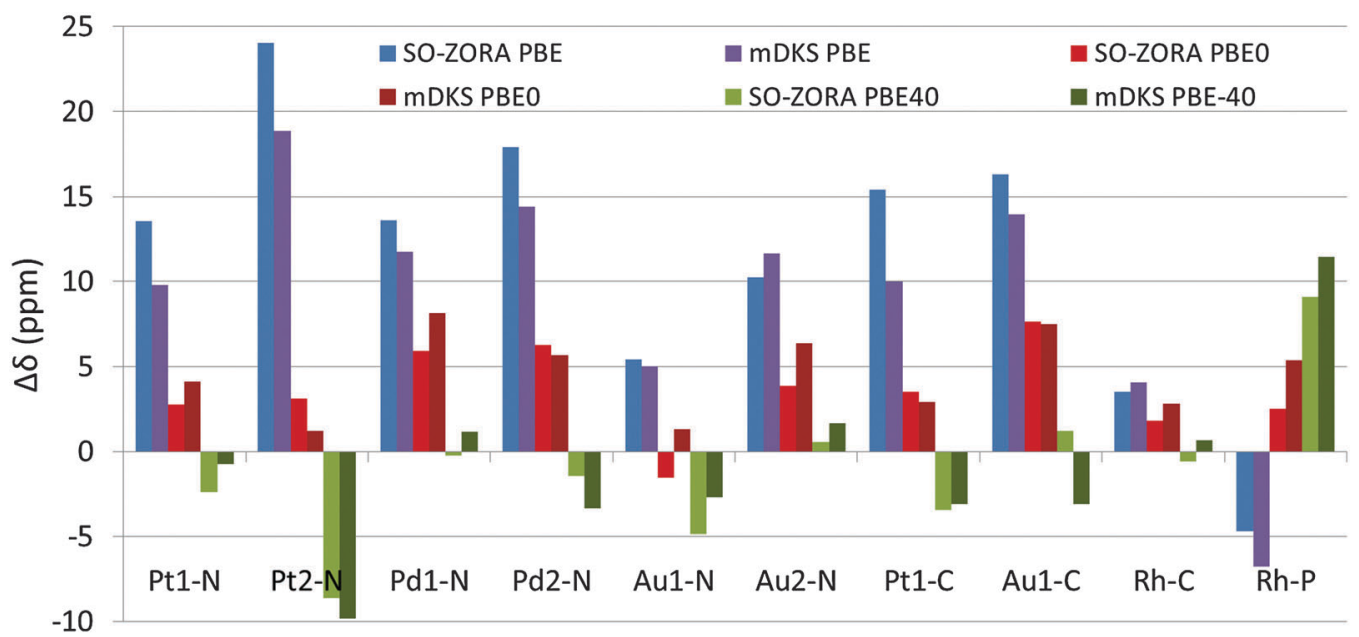

Fig. 7 NMR chemical shifts calculated using $2 \mathrm{c}$ SO-ZORA and $4 \mathrm{c}$ mDKS methods at various levels of theory with solvent correction $\left(\Delta \delta_{\text {solv }}\right)$. Results are reported as standard deviation from the experimental value. 


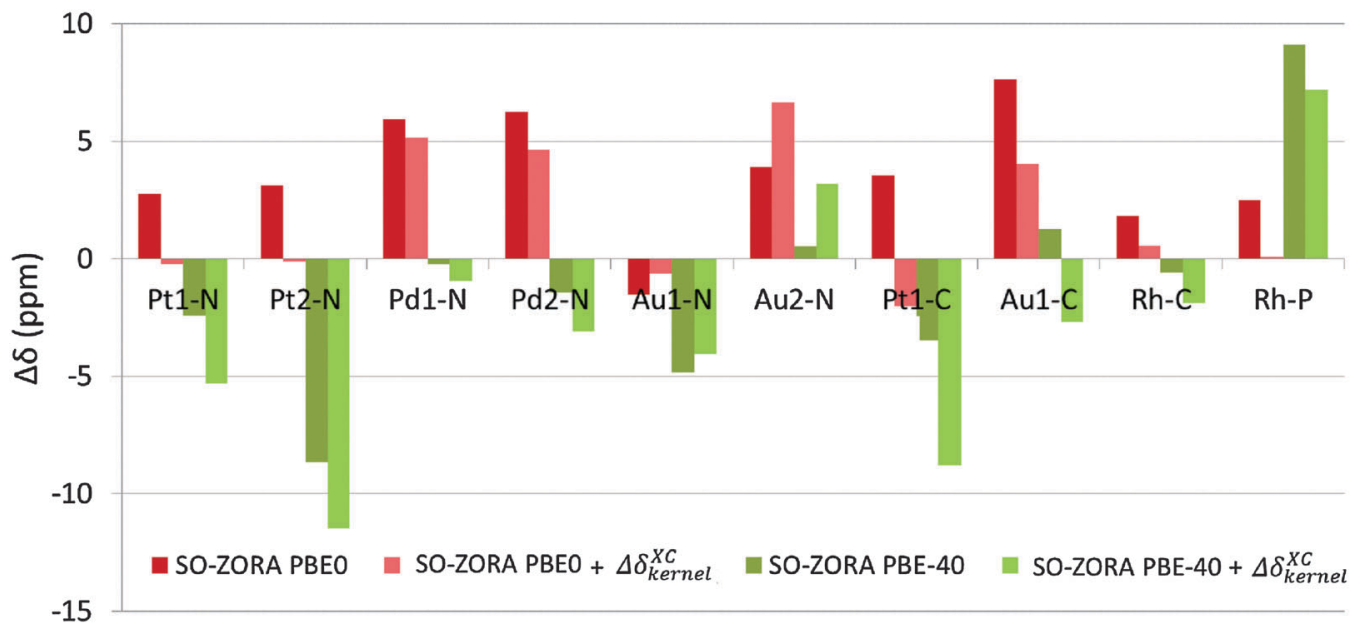

Fig. 8 The NMR chemical shifts calculated at the $2 c$ level using PBEO (red) and PBE-40 (green) functionals (a) without $\Delta \delta_{\mathrm{kernel}}^{\mathrm{XC}}$ contribution (dark colors) and (b) with the addition of $\Delta \delta_{\text {kernel }}^{\mathrm{XC}}$ values (light colors).

difference between the full mDKS and that without SO. Analogously to $\Delta \delta_{\text {solv }}$, the shielding character of $\Delta \delta_{\text {so }}$ is increasing considerably with increasing EE admixture, see Table 4.

The increase in the shielding character of $\Delta \delta_{\mathrm{so}}$ is an important factor for explaining the differences between the values calculated at the PBE, PBE0, and PBE-40 levels (vide infra). Comparing $\Delta \delta_{\text {so }}$ calculated at these three levels of theory with total NMR chemical shift differences (in vacuo, Table S1, ESI $\dagger$ ) indicates that $\Delta \Delta \delta_{\text {so }}$ (Table 4 ) induced by altering the exact-exchange admixture $(40 \rightarrow 0 \%)$ can represent a significant portion of the $\Delta \Delta \delta_{\text {total }}$.

The $\Delta \delta_{\text {SO }}$ values in Table 4 calculated using the ZORA and mDKS methods are of a comparable magnitude. Generally, the $\Delta \delta_{\text {so }}$ values vary between $+5 \mathrm{ppm}$ for Au2 (deshielding) and $-30 \mathrm{ppm}$ for Pt2 (strong shielding contribution). It should be noted that the electronic factors responsible for the SO deshielding (Au2-N) or small $\Delta \delta_{\text {So }}$ (Au1-N) in the gold complexes compared to their platinum counterparts were interpreted very recently by our group as a result of the involvement of $6 \mathrm{p}$ orbitals in $\mathrm{Au}-\mathrm{N}$ bonding ${ }^{82}$ caused by the electrostatic potential of $\mathrm{Au}^{3+}$ and large scalar-relativistic effects ${ }^{90}$ found in the gold complexes.

\subsection{The effect of response exchange-correlation kernel}

The kernel-corrected SO-ZORA approach for NMR CS calculations is currently missing from the available code (ADF 2014). Therefore, we are using the $4 \mathrm{c} \mathrm{mDKS}$ approach at the PBE, PBE0, and PBE-40 levels to estimate an approximate influence of terms arising from the missing XC response kernel $\left(f_{\mathrm{xc}}\right)$ to the NMR chemical shift (see Table S3 in ESI $\dagger$ ), denoted as $\Delta \delta_{\mathrm{kernel}}^{\mathrm{XC}}$. The $\Delta \delta_{\mathrm{kernel}}^{\mathrm{XC}}$ was found to range between +0.3 and $-5.2 \mathrm{ppm}$, with an average value of about $-2.1 \mathrm{ppm}$ for PBE and between +2.7 and $-5.3 \mathrm{ppm}(-1.8 \mathrm{ppm}$ in average) for PBE40. Similarly to $\Delta \delta_{\mathrm{so}},{ }^{82}$ the $\Delta \delta_{\mathrm{kernel}}^{\mathrm{XC}}$ values cause deshielding in $\mathrm{Au}$ complexes, but they have shielding character for the Pt, Pd, and $\mathrm{Rh}$ compounds (see Table S3, ESI $\dagger$ ). On average, the exchange-correlation kernel corresponds to $15-20 \%$ of $\Delta \delta_{\text {so }}$ found for a given spectator atom.
Table 5 Difference between $\Delta \delta_{\text {SO }}$ calculated using the PBE-40 and PBEO functionals at the 2c SO-ZORA level $\left(\Delta \Delta \delta_{S O}\right) . \Delta \delta_{\mathrm{kernel}}^{\mathrm{XC}}$ for the PBEO level calculated using the $4 \mathrm{c}$ mDKS approach

\begin{tabular}{llc}
\hline Atom & $\Delta \Delta \delta_{\text {SO }}{ }^{a}$ & $\Delta \delta_{\text {kernel }}^{\mathrm{XC}}$ \\
\hline Pt1-N & -3.9 & -3.0 \\
Pt2-N & -2.8 & -3.2 \\
Pd1-N & -3.1 & -0.8 \\
Pd2-N & -3.6 & -1.6 \\
Au1-N & -1.0 & 0.9 \\
Au2-N & -0.6 & 2.7 \\
Pt1-C & -5.9 & -5.6 \\
Au1-C & -4.1 & -3.6 \\
Rh-C & -1.6 & -1.3 \\
Rh-P & -2.9 & -2.4 \\
\end{tabular}

${ }^{a}$ Calculated at the SO-ZORA level as $\Delta \Delta \delta_{\mathrm{SO}}=\Delta \delta_{\mathrm{PBE} 0}^{\mathrm{SO}}-\Delta \delta_{\mathrm{PBE}-40 \cdot}^{\mathrm{SO}}{ }^{b} \Delta \delta_{\mathrm{kernel}}^{\mathrm{XC}}$ estimated at the mDKS level by using the PBE0 functional.

To illustrate the role of the missing $f_{\mathrm{xc}}$ terms, appropriate $\Delta \delta_{\mathrm{kernel}}^{\mathrm{XC}}$ values were added to the 2c SO-ZORA NMR chemical shifts calculated at the PBE0 and PBE-40 levels, see Fig. 8.

Upon addition of $\Delta \delta_{\mathrm{kernel}}^{\mathrm{XC}}$, the majority of the PBE0 results were improved, with the RMSD ${ }_{\mathrm{PBEO}}^{\mathrm{SO}-\mathrm{ZORA}}$ reduced to $3.4 \mathrm{ppm}$, whereas the PBE-40 results are uniformly deteriorated $\left(\mathrm{RMSD}_{\mathrm{PBE}-40}^{\mathrm{SO} O \mathrm{RA}}=\right.$ $5.8 \mathrm{ppm}$ ). The most important fact is revealed, however, when the differences in $\Delta \delta_{\text {So }}$ caused by the increase of the $\mathrm{EE}$ admixture from $25 \%$ to $40 \%$ are compared with the $\Delta \delta_{\mathrm{kernel}}^{\mathrm{xC}}$ calculated at the PBE0 level, see Table 5. Our observations suggest that the absence of the response $f_{\mathrm{xc}}$ term in the current SO-ZORA implementation (ADF2014) results in $\Delta \delta_{\text {kernel }}^{\mathrm{XC}}$ deviations that are almost perfectly counterbalanced by the artificial $\Delta \delta_{\text {so }}$ rise introduced by increasing the EE admixture in the functional $\left(25 \rightarrow 40 \%\right.$ in PBE0), $\Delta \Delta \delta_{\text {so }}$ in Table 5.

\subsection{A summary: importance of the individual effects on the total NMR CS}

To demonstrate the importance of the individual effects on the total NMR CS, we performed a comparative stepwise analysis. This includes the evaluation of the role of geometry, solvent, 


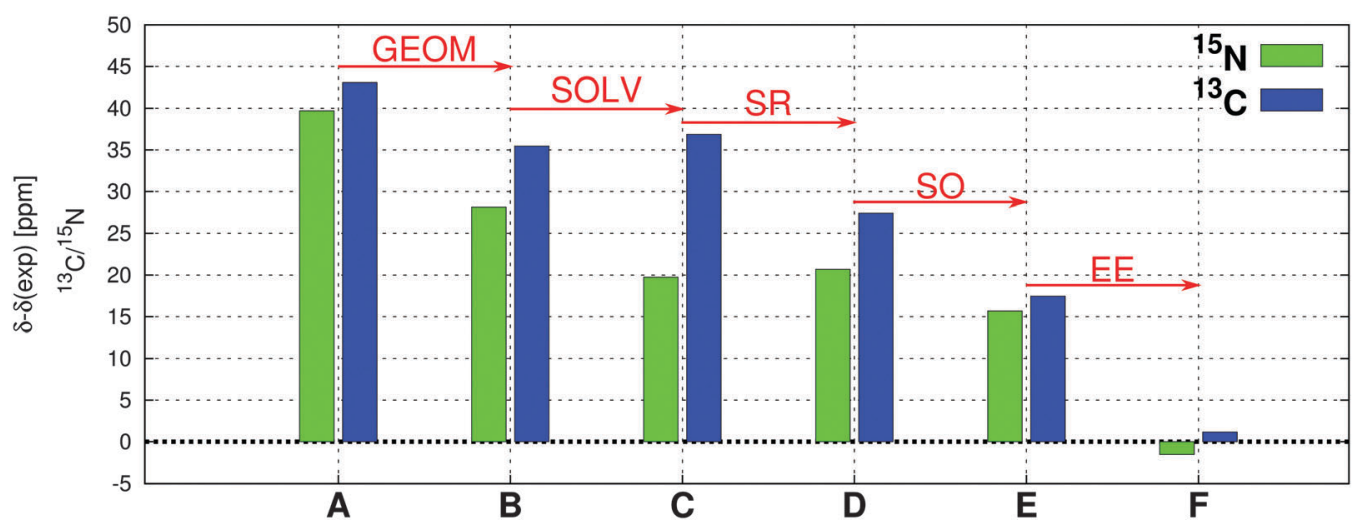

Fig. 9 The deviations of calculated ${ }^{13} \mathrm{C}$ (blue) and ${ }^{15} \mathrm{~N}$ (green) NMR CS from experimental values for compound Au1. The geometry of Au1 was optimized using PBEO/def2-TZVPP/def2-ECP/COSMO DMSO $_{\text {with an exception of A where the BLYP/def2-TZVPP/def2-ECP/COSMO }}$ DMSO approach was used. The NMR chemical shifts were calculated in ADF at various levels of theory: (A) PBE/TZP, (B) PBE/TZP, (C) PBE/TZP/COSMODMSO, (D) PBE/TZP/ZORA/ COSMO $_{\text {DMSO, }}$ (E) PBE/TZP/SO-ZORA/COSMO DMSO, and (F) PBE-40/TZP/SO-ZORA/COSMO DMSO. The differences between neighboring columns represent the effects on the NMR chemical shifts of geometry (GEOM), solvent (SOLV), scalar relativity (SR), spin-orbit coupling (SO), and $40 \%$ admixture of exact exchange in the PBE functional (EE).

scalar relativity, spin-orbit coupling, and admixture of exact exchange in the functional as discussed in detail in Sections 3.1-3.5. The case study of ${ }^{13} \mathrm{C}$ and ${ }^{15} \mathrm{~N}$ NMR chemical shifts for compound Au1 is shown in Fig. 9 (for additional data, see ESI $\dagger$ ).

Although individual factors play different roles in ${ }^{13} \mathrm{C}$ and

${ }^{15} \mathrm{~N}$ NMR CS of different compounds, the EE admixture seems to be crucial and one of the most important factors for reproducing the experimental NMR values. However, as demonstrated here, when the correct geometry and the treatment of solvent effects is combined with the proper description of relativity (including terms arising from $f_{\mathrm{xc}}$ ), standard $25 \%$ exact-exchange admixture in the PBE0 functional (instead of somewhat artificial 40\%) seems to be sufficient for the NMR CS calculations in squareplanar transition-metal complexes.

\section{Conclusions}

In this work, we evaluated the effects of molecular geometry, implicit solvent, role of the functional, and the treatment of relativistic effects on the NMR chemical shifts calculated for light atoms in the vicinity of a heavy-metal center in selected square-planar complexes. The selection of the DFT functional used for optimizing the geometry was performed by a cluster approach. The best geometries were obtained for the PBE0 functional, closely followed by TPSSH and MN12SX. The effect of geometry on the NMR chemical shifts (deviation between best and worst structure) was found to be rather large, $14 \mathrm{ppm}$ for ${ }^{15} \mathrm{~N}$ and $22 \mathrm{ppm}$ for ${ }^{13} \mathrm{C}$.

The role of an implicit solvent was found to be of considerable importance for the NMR chemical shifts in the square-planar complexes, mainly for ${ }^{15} \mathrm{~N}$, with an average $13 \mathrm{ppm}$ contribution to the total NMR chemical shifts (estimated at the SO-ZORA level using the PBE0 functional).

The most important relativistic NMR CS contribution is the $\Delta \delta_{\text {so, }}$, with its size varying between $+5 \mathrm{ppm}$ and $-30 \mathrm{ppm}$ (for both 2c SO-ZORA and 4c mDKS), which was also demonstrated to be highly dependent on the amount of exact-exchange admixture in the functional.

The importance of increased exact-exchange admixture to $40 \%$ in the PBE0 functional for the 2c SO-ZORA approach was confirmed for the light spectator atoms $\left({ }^{13} \mathrm{C},{ }^{15} \mathrm{~N}\right.$, and $\left.{ }^{31} \mathrm{P}\right)$, and rationalized as a compensation for the missing $\Delta \delta_{\mathrm{kernel}}^{\mathrm{XC}}$ contribution. Therefore, with the current implementation of the SO-ZORA approach used in this work, the PBE-40 functional slightly outperforms PBE0. However, accounting for the effect of the response exchangecorrelation kernel, the standard PBE0 functional provides somewhat better agreement with the experiment. Undoubtedly, the effect of the XC kernel should not be neglected for systems with a large HALA effect, such as actinides. ${ }^{7}$

Finally, the relative importance of individual effects (geometry, solvent, relativistic approximation, DFT functional) to the total NMR CS is demonstrated by stepwise calculations. Currently, the use of hybrid GGA functionals (with the DFT functionals used) is mandatory for reproducing the experimental NMR data in heavy-element compounds.

In the fully relativistic mDKS approach using restricted magnetically balanced basis sets, an implementation of GIAOs for hybrid functionals as well as an implicit solvent model is still missing. However, using simple empirical corrections estimated at the 2c SO-ZORA level provides reliable NMR CS data at affordable computational costs. Undoubtedly, the fourcomponent mDKS method would outperform $2 \mathrm{c}$ approaches in calculating heavy-element NMR parameters. ${ }^{91}$ Our efforts in this direction will be reported elsewhere.

\section{Acknowledgements}

This work was supported by the Czech Science Foundation (15-09381S to RM and 14-03564S to MS) and carried out at CEITEC - the Central European Institute of Technology with research infrastructure supported by the project CZ.1.05/1.1.00/ 02.0068 financed by the European Regional Development Fund. 
This work also received support from the Research Council of Norway through a Centre of Excellence Grant and project grants (Grant No. 179568, 214095 and 177558). The Czech-Norway mobility grant (NF-CZ07-MOP-3-245-2015) from Norway Funds administrated by Ministry of Education of the Czech Republic is gratefully acknowledged. The access to computing and storage facilities owned by parties and projects contributing to the National Grid Infrastructure MetaCentrum, and provided under the program "Projects of Large Infrastructure for Research, Development, and Innovations" (LM2010005), and the CERIT-SC computing and storage facilities, provided under the program Center CERIT Scientific Cloud, part of the Operational Program Research and Development for Innovations (CZ.1.05/3.2.00/08.0144), is acknowledged.

\section{References}

1 X. Wang and Z. Guo, Chem. Soc. Rev., 2012, 42(1), 202-224.

2 D. Steinborn, Fundamentals of Organometallic Catalysis, in Translator, ed. A. Frankel, Wiley-VCH, Weinheim, 1st edn, 2011.

3 I. Eryazici, C. N. Moorefield and G. R. Newkome, Chem. Rev., 2008, 108(6), 1834-1895.

4 J. Vícha, G. Demo and R. Marek, Inorg. Chem., 2012, 51(3), 1371-1379.

5 N. Nakagawa, S. Coelho and S. Obinata, 6th NMR Symp. 1967, p. 8.

6 M. Kaupp, O. L. Malkina, V. G. Malkin and P. Pyykkö, Chem. - Eur. J., 1998, 4(1), 118-126.

7 P. Hrobárik, V. Hrobáriková, A. H. Greif and M. Kaupp, Angew. Chem., Int. Ed., 2012, 51(43), 10884-10888.

8 M. Bühl and H. Kabrede, J. Chem. Theory Comput., 2006, 2(5), 1282-1290.

9 J. Vícha, M. Straka, M. L. Munzarová and R. Marek, J. Chem. Theory Comput., 2014, 10(4), 1489-1499.

10 J. Vícha, M. Patzschke and R. Marek, Phys. Chem. Chem. Phys., 2013, 15(20), 7740-7754.

11 T. Pawlak, M. L. Munzarová, L. Pazderski and R. Marek, J. Chem. Theory Comput., 2011, 7(12), 3909-3923.

12 M. Bühl, C. Reimann, D. A. Pantazis, T. Bredow and F. Neese, J. Chem. Theory Comput., 2008, 4(9), 1449-1459.

13 P. Comba, in Fundamental Principles of Molecular Modeling, ed. W. Gans, A. Amann and J. C. A. Boeyens, Springer, US, 1996, pp. 167-187.

14 P. Comba, in Intermolecular Interactions, ed. W. Gans and J. C. A. Boeyens, Springer, US, 1998, pp. 97-109.

15 T. Pawlak, D. Niedzielska, J. Vícha, R. Marek and L. Pazderski, J. Organomet. Chem., 2014, 759, 58-66.

16 A. Klamt and G. Schüürmann, J. Chem. Soc., Perkin Trans. 2, 1993, 799-805.

17 S. Standara, P. Kulhánek, R. Marek, J. Horníček, P. Bouř and M. Straka, Theor. Chem. Acc., 2011, 129(3-5), 677-684.

18 L. A. Truflandier, K. Sutter and J. Autschbach, Inorg. Chem., 2011, 50(5), 1723-1732.

19 K. Aidas, A. Møgelhøj, C. B. Nielsen, K. V. Mikkelsen, K. Ruud, O. Christiansen and J. Kongsted, J. Phys. Chem. A, 2007, 111(20), 4199-4210.
20 M. Dračínský and P. Bouř, J. Chem. Theory Comput., 2010, 6(1), 288-299.

21 J. M. Olsen, K. Aidas and J. Kongsted, J. Chem. Theory Comput., 2010, 6(12), 3721-3734.

22 F. Jensen, Introduction to Computational Chemistry, Wiley, 2006.

23 T. Saue, ChemPhysChem, 2011, 12(17), 3077-3094.

24 E. van Lenthe, J. G. Snijders and E. J. Baerends, J. Chem. Phys., 1996, 105(15), 6505-6516.

25 L. A. Seaman, P. Hrobárik, M. F. Schettini, S. Fortier, M. Kaupp and T. W. Hayton, Angew. Chem., Int. Ed., 2013, 52(11), 3259-3263.

26 K. Sutter and J. Autschbach, J. Am. Chem. Soc., 2012, 134(32), 13374-13385.

27 M. Bühl, Chem. Phys. Lett., 1997, 267(3-4), 251-257.

28 J. Autschbach, Mol. Phys., 2013, 111(16-17), $2544-2554$.

29 K. G. Dyall and K. Faegri Jr, Introduction to Relativistic Quantum Chemistry, Oxford University Press, 2007, pp. 35-53.

30 S. Komorovsky, M. Repisky, K. Ruud, O. L. Malkina and V. G. Malkin, J. Phys. Chem. A, 2013, 117(51), 14209-14219.

31 L. Belpassi, L. Storchi, H. M. Quiney and F. Tarantelli, Phys. Chem. Chem. Phys., 2011, 13(27), 12368-12394.

32 S. Komorovský, M. Repiský, O. L. Malkina, V. G. Malkin, I. Malkin Ondík and M. Kaupp, J. Chem. Phys., 2008, 128(10), 104101.

33 S. Komorovský, M. Repiský, O. L. Malkina and V. G. Malkin, J. Chem. Phys., 2010, 132(15), 154101.

34 S. Komorovsky, M. Repisky, E. Malkin, T. B. Demissie and K. Ruud, J. Chem. Theory Comput., 2015, 11(8), 3729-3739.

35 M. Kobayashi, S. Masaoka and K. Sakai, Acta Crystallogr., Sect. E: Struct. Rep. Online, 2008, 64(10), m1325.

36 L. Pazderski, T. Pawlak, J. Sitkowski, L. Kozerski and E. Szłyk, Magn. Reson. Chem., 2009, 47(11), 932-941.

37 M. A. Bruck, R. Bau, M. Noji, K. Inagaki and Y. Kidani, Inorg. Chim. Acta, 1984, 92(4), 279-284.

38 Y. Muranishi, Y. Wang, M. Odoko and N. Okabe, Acta Crystallogr., Sect. C: Cryst. Struct. Commun., 2005, 61(6), m307-m310.

39 L. Pazderski, J. Toušek, J. Sitkowski, L. Kozerski and E. Szłyk, Magn. Reson. Chem., 2007, 45(12), 1059-1071.

40 E. J. MacLean, R. I. Robinson, S. J. Teat, C. Wilson and S. Woodward, J. Chem. Soc., Dalton Trans., 2002, 3518-3524.

41 T. Pawlak, L. Pazderski, J. Sitkowski, L. Kozerski and E. Szłyk, Magn. Reson. Chem., 2011, 49(2), 59-64.

42 D. Niedzielska, T. Pawlak, T. Czubachowski and L. Pazderski, J. Spectrosc., 2012, 2013, 1-8.

43 Y. Fuchita, H. Ieda, Y. Tsunemune, J. Kinoshita-Nagaoka and H. Kawano, J. Chem. Soc., Dalton Trans., 1998, 791-796.

44 X.-P. Zhang, G. Yang, L. Wang and S. W. Ng, Acta Crystallogr., Sect. E: Struct. Rep. Online, 2007, 63(6), m1582.

45 L. Pazderski, J. Toušek, J. Sitkowski, L. Kozerski and E. Szłyk, Magn. Reson. Chem., 2009, 47(8), 658-665.

46 E. Kossoy, B. Rybtchinski, Y. Diskin-Posner, L. J. W. Shimon, G. Leitus and D. Milstein, Organometallics, 2009, 28(2), 523-533.

47 K. Maliňáková, L. Novosadová, M. Lahtinen, E. Kolehmainen, J. Brus and R. Marek, J. Phys. Chem. A, 2010, 114(4), 1985-1995. 
48 K. Maliňáková, L. Novosadová, M. Pipíška and R. Marek, ChemPhysChem, 2011, 12(2), 379-388.

49 K. Bouzková, M. Babinský, L. Novosadová and R. Marek, J. Chem. Theory Comput., 2013, 9(6), 2629-2638.

50 M. Babinský, K. Bouzková, M. Pipíška, L. Novosadová and R. Marek, J. Phys. Chem. A, 2013, 117(2), 497-503.

51 F. Weigend and R. Ahlrichs, Phys. Chem. Chem. Phys., 2005, 7(18), 3297-3305.

52 D. Andrae, U. Häußermann, M. Dolg, H. Stoll and H. Preuß, Theor. Chim. Acta, 1990, 77(2), 123-141.

53 C. Lee, W. Yang and R. G. Parr, Phys. Rev. B, 1988, 37(2), 785-789.

54 A. D. Becke, Phys. Rev. A: At., Mol., Opt. Phys., 1988, 38(6), 3098-3100.

55 P. J. Stephens, F. J. Devlin, C. F. Chabalowski and M. J. Frisch, J. Phys. Chem., 1994, 98(45), 11623-11627.

56 A. D. Becke, J. Chem. Phys., 1993, 98(2), 1372-1377.

57 J. Paier, M. Marsman and G. Kresse, J. Chem. Phys., 2007, $127(2), 024103$.

58 T. Yanai, D. P. Tew and N. C. Handy, Chem. Phys. Lett., 2004, 393(1-3), 51-57.

59 J. P. Perdew, K. Burke and M. Ernzerhof, Phys. Rev. Lett., 1996, 77(18), 3865-3868; J. P. Perdew, K. Burke and M. Ernzerhof, Phys. Rev. Lett., 1997, 78, 1396.

60 J. P. Perdew, Phys. Rev. B: Condens. Matter Mater. Phys., 1986, 33(12), 8822-8824; J. P. Perdew, Phys. Rev. B: Condens. Matter Mater. Phys., 1986, 34, 7406.

61 V. N. Staroverov, G. E. Scuseria, J. Tao and J. P. Perdew, J. Chem. Phys., 2003, 119(23), 12129-12137.

62 J. P. Perdew, J. Tao, V. N. Staroverov and G. E. Scuseria, J. Chem. Phys., 2004, 120(15), 6898-6911.

63 C. Adamo and V. Barone, J. Chem. Phys., 1999, 110(13), 6158-6170.

64 C. Adamo, G. E. Scuseria and V. Barone, J. Chem. Phys., 1999, 111(7), 2889-2899.

65 S. Grimme, J. Comput. Chem., 2006, 27(15), 1787-1799.

66 J.-D. Chai and M. Head-Gordon, Phys. Chem. Chem. Phys., 2008, 10(44), 6615-6620.

67 Y. Minenkov, Å. Singstad, G. Occhipinti and V. R. Jensen, Dalton Trans., 2012, 41(18), 5526-5541.

68 R. Peverati and D. G. Truhlar, Phys. Chem. Chem. Phys., 2012, 14(47), 16187-16191.

69 S. Grimme, J. Comput. Chem., 2004, 25(12), 1463-1473.

70 E. van Lenthe, E. J. Baerends and J. G. Snijders, J. Chem. Phys., 1994, 101(11), 9783-9792.
71 E. van Lenthe, E. J. Baerends and J. G. Snijders, J. Chem. Phys., 1993, 99(6), 4597-4610.

72 K. Wolinski, J. F. Hinton and P. Pulay, J. Am. Chem. Soc., 1990, 112(23), 8251-8260.

73 M. Repiský, S. Komorovský, V. G. Malkin, O. L. Malkina, M. Kaupp, K. Ruud, R. Bast, U. Ekstrom, M. Kadek, S. Knecht, L. Konečný, E. Malkin and I. Malkin Ondík, Relativistic Spectroscopy DFT program ReSpect, developer version 3.4.2, 2015, www.respectprogram.org.

74 F. Jensen, J. Chem. Phys., 2002, 116(8), 3502.

75 F. Jensen and T. Helgaker, J. Chem. Phys., 2004, 121(8), 3463-3470.

76 K. G. Dyall, Theor. Chem. Acc., 2004, 112(5-6), 403-409.

77 K. G. Dyall, Theor. Chem. Acc., 2006, 117(4), 483-489.

78 K. G. Dyall and A. S. P. Gomes, Theor. Chem. Acc., 2009, 125(1-2), 97-100.

79 R. Marek and A. Lycka, Curr. Org. Chem., 2002, 6(1), 35-66. 80 R. Marek and A. Lyčka, Curr. Org. Chem., 2007, 11, 1154-1205. 81 A. Bruker, Bruker GmbH, Karlsruhe, Germany, 1991.

82 J. Vícha, C. Foroutan-Nejad, T. Pawlak, M. L. Munzarová, M. Straka and R. Marek, J. Chem. Theory Comput., 2015, 11(4), 1509-1517.

83 J. Toušek, S. Van Miert, L. Pieters, G. Van Baelen, S. Hostyn, B. U. W. Maes, G. Lemière, R. Dommisse and R. Marek, Magn. Reson. Chem., 2008, 46(1), 42-51.

84 R. Marek, in Encyclopedia of Spectroscopy and Spectrometry, ed. J. C. Lindon, Academic Press, Oxford, 2nd edn, 2010, pp. 1892-1898.

85 H. Andersson, A.-C. C. Carlsson, B. Nekoueishahraki, U. Brath and M. Erdélyi, Annual Reports on NMR Spectroscopy, Academic Press, 2015, DOI: 10.1016/bs.arnmr.2015.04.002.

86 S. Standara, K. Maliňáková, R. Marek, J. Marek, M. Hocek, J. Vaara and M. Straka, Phys. Chem. Chem. Phys., 2010, 12(19), 5126-5139.

87 P. Manninen, P. Lantto, J. Vaara and K. Ruud, J. Chem. Phys., 2003, 119(5), 2623-2637.

88 J. Vaara, P. Manninen and P. Lantto, in Calculation of NMR and EPR Parameters, ed. M. Kaupp, M. Bühl and V. Malkin, Wiley-VCH, Verlag GmbH \& Co. KGaA, 2004, pp. 209-226.

89 P. Manninen, K. Ruud, P. Lantto and J. Vaara, J. Chem. Phys., 2005, $122(11), 114107$.

90 P. Pyykko and J. P. Desclaux, Acc. Chem. Res., 1979, 12(8), 276-281.

91 E. Malkin, S. Komorovsky, M. Repisky, T. B. Demissie and K. Ruud, J. Phys. Chem. Lett., 2013, 4(3), 459-463. 